3 Moore VC, Jaakkola MS, Burge CB, et al. A new diagnostic score for occupational asthma. The area between the curves (ABC score) of peak expiratory flow on days at and away from work. Chest 2009; 135: 307-314.

4 Yan K, Salome C, Woolcock AJ. Rapid method for measurement of bronchial responsiveness. Thorax 1983; 38: 760-765.
5 Jarvis J, Seed MJ, Elton R, et al. Relationship between chemical structure and the occupational asthma hazard of low molecular weight organic compounds. Occup Environ Med 2005; 62 243-250.

\title{
Response to salbutamol in patients with mild asthma treated with nadolol
}

\section{To the Editors:}

$\beta$-blockers (inverse agonists) have an exactly opposite effect to $\beta_{2}$-adrenoceptor $\left(\beta_{2}\right.$-ADR) agonists and are currently contraindicated in asthma. This results from the fact that the acute administration of these drugs can produce bronchoconstriction by blocking the bronchodilating effects of endogenous adrenaline, or by inactivating constitutively active $\beta_{2}$-ADRs, and can worsen asthma symptoms [1]. In fact, published accounts have shown that acute $\beta$-blocker use can, but will not always, precipitate airway constriction in patients with asthma [2]. Nevertheless, there are compelling uses of $\beta$-blockers in asthmatics, such as in heart failure and after myocardial infarction. The effect of chronic administration of $\beta$-blockers in asthma has remained unknown until recently, when studies on a murine model of asthma showed that, while acute (singledose) administration of $\beta$-blockers increased airway hyperresponsiveness (AHR), their chronic (28-day) administration had an opposite effect and decreased AHR [3]. Furthermore, recent data demonstrate that chronic $\beta$-blocker treatment produces broad anti-inflammatory effects, and especially dramatic effects on airway epithelium and mucous metaplasia [4, 5]. More recently, our group has further confirmed the pivotal role that $\beta_{2}$-ADR has on mucous metaplasia using a $\beta_{2}$-ADR null mouse [6]. Based on these findings in the murine asthma model, we investigated the role of chronic administration of nadolol (a non-selective $\beta_{1}$ - and $\beta_{2}$-blocker) on airway hyperresponsiveness in an open-label study of 10 subjects with mild asthma [7]. We demonstrated that nadolol produced a dose-dependent increase in the PC20 (provocative dose causing a $20 \%$ fall in forced expiratory volume in $1 \mathrm{~s}$ (FEV1)) methacholine when administered over 11 weeks. However, a concern that remained is whether these subjects treated with nadolol can still respond to short-acting $\beta_{2}$-ADR agonists for rescue of bronchospasm [8]. In fact, one concern is that nadolol "may block bronchodilation produced by endogenous or exogenous catecholamine stimulation of $\beta_{2}$ receptors" (Corgard ${ }_{\circledR}$ product insert; King Pharmaceuticals, Inc., Bristol, TN, USA).

We have now completed our second open-label study of chronic nadolol in subjects with mild asthma who are not on controller therapy $(n=10)$ (table 1$)$. Both studies were approved by the Baylor College of Medicine Institutional Review Board (Houston, TX, USA) and informed consent was obtained from all subjects. Starting at a daily dose of $1.25 \mathrm{mg}$ and ending at a maximum daily dose of $40 \mathrm{mg}$, nadolol dose was escalated on a biweekly basis based on predetermined safety, lung function, asthma control and haemodynamic parameters. Subjects were treated for a total of 13 weeks with nadolol ( $\geqslant 3$ weeks on their final tolerated dose). Seven out of the 10 subjects were able to be escalated to $40 \mathrm{mg}$, one tolerated

\section{TABLE 1 Baseline demographics and characteristics of study population}

\begin{tabular}{|c|c|c|c|c|c|c|}
\hline Subject no. & Sex & Age yrs & Race & Baseline FEV 1 \% & Baseline $\mathrm{PC}_{20}$ & Final dose $\mathrm{mg}$ \\
\hline 1 & $\mathrm{~F}$ & 48 & Black & 84 & 3.576 & 40 \\
\hline 2 & $\mathrm{~F}$ & 24 & White & 98 & 3.621 & 40 \\
\hline $4^{\#}$ & M & 27 & Hispanic & 81 & 3.479 & 1.25 \\
\hline 5 & $\mathrm{~F}$ & 49 & Hispanic & 86 & 0.112 & 40 \\
\hline 8 & $\mathrm{~F}$ & 26 & Hispanic & 91 & 0.471 & 40 \\
\hline 9 & $\mathrm{~F}$ & 18 & Asian & 83 & 0.372 & 40 \\
\hline 10 & $\mathrm{~F}$ & 29 & White & 114 & 6.418 & 40 \\
\hline Mean \pm SEM & & $32.6 \pm 3.6$ & & $90.7 \pm 3.3$ & $1.18^{\circ} \pm 1.53^{+}$ & $29.6 \pm 6$ \\
\hline
\end{tabular}

${ }^{\#}$ : subject excluded because of an asthma exacerbation early in the study; ${ }^{\bullet}$ : geometric mean; ${ }^{+}$: geometric SEM 


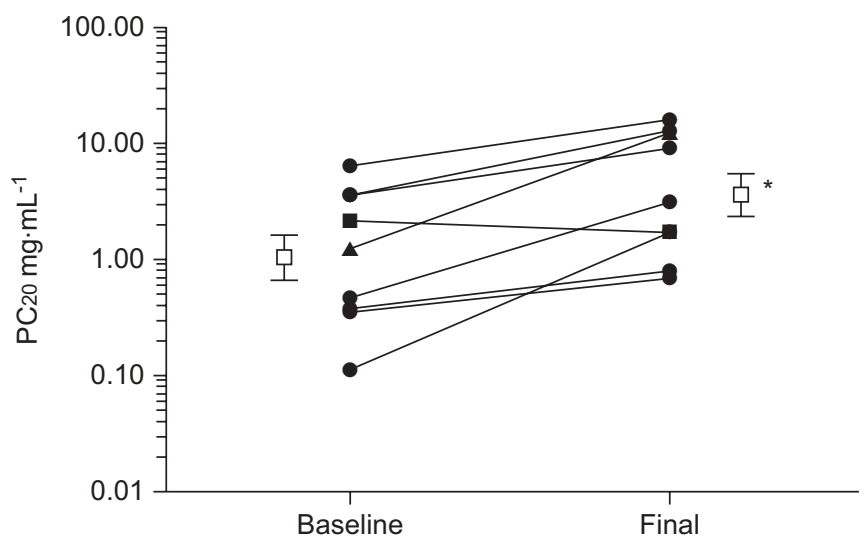

FIGURE 1. The effect of chronic treatment with nadolol on the $\mathrm{PC}_{20}$ (provocative dose causing a $20 \%$ fall in forced expiratory volume in $1 \mathrm{~s}$ ) metacholine in subjects with mild asthma. Data are shown for baseline visit and final visit. Final nadolol doses used for subjects based on dose-escalation are shown. Geometric mean \pm SEM are shown ( $\square$ and whiskers, respectively). $\bullet: 40 \mathrm{mg}$ nadolol $(n=7)$; $\mathbf{\square}: 10 \mathrm{mg}$ nadolol $(n=1) ; \mathbf{\Lambda}: 5 \mathrm{mg}$ nadolol $(n=1)$. Comparisons between $\mathrm{V} 0$ and $\mathrm{V} 10$ values were performed using paired t-tests. ${ }^{*}: \mathrm{p}<0.05$.

a 10-mg daily dose and one tolerated a 5-mg daily dose. One subject was excluded from the study because of an asthma exacerbation early in the study while on a dose of $1.25 \mathrm{mg}$. As demonstrated in our first study [8], we were able to show a significant effect on airway hyperresponsiveness with chronic nadolol dosing (fig. 1) with geometric mean \pm SEM PC20 at baseline and at final visit of $1.04 \pm 1.54$ and $3.61 \pm 1.52$, respectively, and a doubling dose change \pm SEM of $1.79 \pm 0.44$ $(p=0.004)$. No significant changes in lung function were observed (FEV1 \% predicted at baseline and final visit: $91.8 \pm 3.4$ and $87.1 \pm 4.4$, respectively; $p=0.165$ ). To address the effect of nadolol on the bronchodilator response to shortacting $\beta_{2}$-ADR agonists, we evaluated the bronchodilator response after administering salbutamol by nebulisation (2.5 mg) immediately following methacholine challenge performed at the final visit in subjects from both studies $(n=18)$. Spirometry was performed $20 \mathrm{~min}$ following salbutamol administration. As shown in figure 2, salbutamol was able to reverse methacholine-induced bronchoconstriction in all subjects who were on chronic treatment with nadolol.

Our study has several limitations. We studied steroid-naïve patients with mild asthma. In patients with severe disease and inflammation, it is unknown whether chronic nadolol treatment would also show a preserved response to $\beta 2$-ADR agonists. In addition, it is not known whether the observed effects may be seen with the use of lower doses of salbutamol. While we believe that our findings can be explained by the high degree of inverse agonism of nadolol, future work needs to explore this further and compare these effects using $\beta$-blockers with different levels of inverse agonism.

In summary, we demonstrated that in subjects with mild asthma, chronic dosing with an inverse agonist, nadolol, is accompanied by attenuation of airway hyperresponsivess, and administration of salbutamol is able to reverse methacholineinduced bronchoconstriction in these subjects. These findings suggest that $\beta_{2}$-ADRs may not be completely blocked with

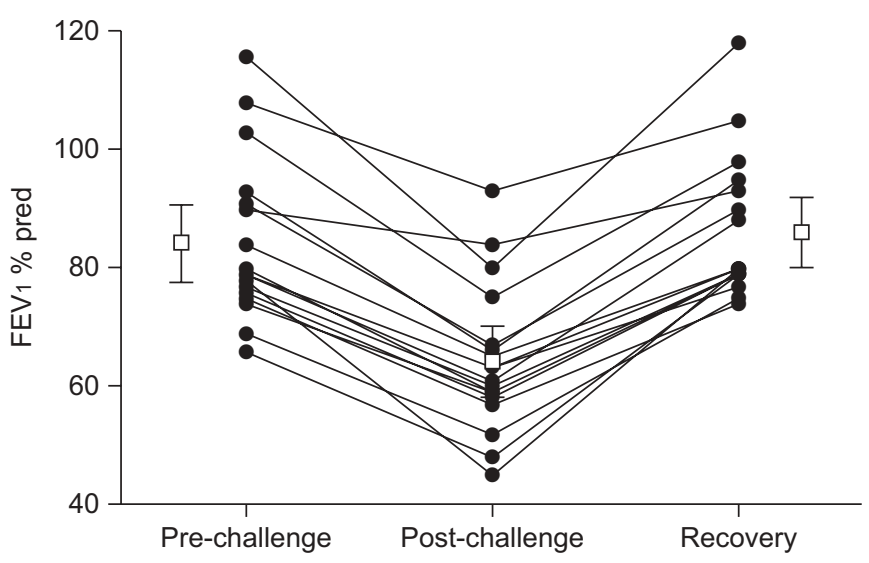

FIGURE 2. Response to salbutamol ( $2.5 \mathrm{mg}$ by nebulisation) administered after metacholine challenge at final visit in subjects with mild asthma treated with nadolol from both studies $(n=18)$. Forced expiratory volume in $1 \mathrm{~s}\left(F \mathrm{FV}_{1}\right) \%$ predicted values pre- and post-challenge and values $20 \mathrm{~min}$ following salbutamol administration (recovery) are shown. Mean values for each time period with $95 \% \mathrm{Cl}$ are also shown ( $\square$ and whiskers, respectively).

chronic nadolol dosing, and are supportive of the data from patients with hypertension and anxiety, which suggested that the use of the $\beta$-blocker propranolol in these populations was associated with increased $\beta_{2}$-ADR bronchodilating function contrary to that which would be expected $[9,10]$. The significance of our findings, if confirmed in larger controlled trials, may provide a paradigm shift in the chronic management of asthma and in the way we view the pathology of asthma.

N.A. Hanania*, B. Mannava*, A.E. Franklin", B.J. Lipworth", P.A. Williamson ${ }^{\oplus}$, W.J. Garner ${ }^{\#}$, B.F. Dickey ${ }^{+}$and R.A. Bond ${ }^{\S}$ *Section of Pulmonary and Critical Care Medicine, Baylor College of Medicine, ${ }^{+}$Dept of Pulmonary Medicine, MD Anderson Cancer Center, ${ }^{\S}$ Dept of Pharmaceutical and Pharmacological Sciences, University of Houston, Houston, TX and, "Inverseon Inc., San Francisco, CA, USA. "Asthma and Allergy Research Group, Ninewells Hospital and Medical School, Dundee, UK.

Correspondence: N.A. Hanania, Section of Pulmonary and Critical Care Medicine, Baylor College of Medicine, 1504 Taub Loop, Houston, TX 77030, USA. E-mail: Hanania@bcm.edu

Support Statement: This work was supported by grants from the National Institutes of Health (NIH; 5K23HL079054) and Sandler's Asthma Research Program.

Statement of Interest: Statements of interest for A.E. Franklin, W.J. Garner and R.A. Bond can be found at www.erj. ersjournals.com/misc/statements.dtl

\section{REFERENCES}

1 Bond RA, Spina D, Parra S, et al. Getting to the heart of asthma: can " $\beta$ blockers" be useful to treat asthma? Pharmacol Ther 2007; 115: 360-374. 
2 Mcneill RS. Effect of a $\beta$-adrenergic-blocking agent, propranolol, on asthmatics. Lancet 1964; 2: 1101-1102.

3 Callaerts-Vegh Z, Evans KL, Dudekula N, et al. Effects of acute and chronic administration of $\beta$-adrenoceptor ligands on airway function in a murine model of asthma. Proc Natl Acad Sci USA 2004; 101: 4948-4953.

4 Lin R, Peng H, Nguyen LP, et al. Changes in $\beta_{2}$-adrenoceptor and other signaling proteins produced by chronic administration of "B-blockers" in a murine asthma model. Pulm Pharmacol Ther 2008; 21: $115-124$.

5 Nguyen LP, Omoluabi O, Parra S, et al. Chronic exposure to beta-blockers attenuates inflammation and mucin content in a murine asthma model. Am J Respir Cell Mol Biol 2008; 38: 256-262.
6 Nguyen LP, Lin R, Parra S, et al. $\beta_{2}$-adrenoceptor signaling is required for the development of an asthma phenotype in a murine model. Proc Natl Acad Sci USA 2009; 106: 2435-2440.

7 Hanania NA, Singh S, El-Wali R, et al. The safety and effects of the $\beta$-blocker, nadolol, in mild asthma: an open-label pilot study. Pulm Pharmacol Ther 2008; 21: 134-141.

8 Lipworth BJ, Williamson PA. $\beta$ blockers for asthma: a doubleedged sword. Lancet 2009; 373: 104-105.

9 Lima DR, Turner P. Propranolol increases reduced $\beta$-receptor function in severely anxious patients. Lancet 1983; 2: 1505.

10 Lima DR, Turner P. $\beta$-blocking drugs increase responsiveness to prostacyclin in hypertensive patients. Lancet 1982; 2: 444.

DOI: 10.1183/09031936.00003210

\section{Endobronchial metallic clips to guide high-dose external-beam radiotherapy in radio-occult lung cancer}

\section{To the Editors:}

As a result of the increased sensitivity of videobronchoscopy (including narrow-band and video autofluorescence imaging) and a specific interest in surveillance of pre-invasive lesions, an increase in diagnosis of radiographically occult lung carcinoma (ROLC) can be anticipated. Described for the first time in the late 1970s by MARTINI and MELAMED [1] and in the early 1980s by CORTESE et al. [2], surgery still remains the treatment of choice for ROLC [3]. However, patients can be functionally inoperable due to comorbidity, advanced age, multiple primary lesions or irresectable, centrally localised tumours, or because they refuse surgery [2]. Therefore, varieties of endoscopic techniques, such as photodynamic therapy, cryotherapy, laser therapy, electrocautery and brachytherapy, were developed as alternatives to surgery [3-5]. The combination of high-dose external-beam radiotherapy (EBRT) with intraluminal radiotherapy (IR) using low [6-8], middle [9] and high dose-rate [10] iridium-192, has been investigated in ROLC with promising results. EBRT has obvious advantages to surgery, as it is less invasive and better tolerated by the patient. A major issue is, however, the localisation of the tumour for the guidance of EBRT, since these tumours are radiologically occult. Previously, in those studies combining EBRT with IR [6-10], localisation of tumour was determined under fluoroscopy using the tip of the bronchoscope. Radiation was given using small anteriorposterior opposed portals. Obviously, this is less reliable, precise and comfortable, as might be anticipated. To overcome this problem, we hypothesised that metallic clips, the same as used in gastroenterology to obtain haemostasis, could be used as beacons to locate the tumour during EBRT. In a postoperative setting, the same principle is often used to orient EBRT in incomplete resections. Radiation fields are collimated using metallic clips placed by the surgeon.

In a prospective pilot series (June-October 2008) we assessed the feasibility and safety of EZ-Clips HX 610-090 (Olympus, Aartselaar, Belgium) in inoperable patients with ROLC to guide high-dose EBRT. The standard length of these clips is $6 \mathrm{~mm}$ when deployed, and when opened the angle of the jaws is $90^{\circ}$ (fig. 1). Patient characteristics and tumour locations are summarised in table 1 . All four patients were male, smokers with a median age of 68 yrs and fitted the criteria for ROLC as described by the Japan Society of Lung Cancer [8]. They underwent white light and autofluorescence video bronchoscopy (fig. 1) because of haemoptysis (case 1), persistent infiltrate (case 2), screening for second primary (case 3) and persistant cough (case 4). Pathology of the endobronchial biopsies was invasive squamous cell carcinoma for all patients. One was staged cT2N0M0 and three were cT1N0M0. Staging was performed according to the 6th edition of the TNM (tumour, nodes, metastasis) Classification of Malignant Tumours [11]. Patients 1, 2 and 4 received brachytherapy with one fraction of $10 \mathrm{~Gy}$ at $1 \mathrm{~cm}$ from the catheter. Patient $3 \mathrm{did}$ not receive endobronchial brachytherapy because of a concurrent supraglottic tumour. Before EBRT, the patients underwent a second bronchoscopy to place two endobronchial metallic clips: one on the proximal carina and one on the distal carina, relative to the ROLC (fig. 1). This was performed under local anaesthesia and was well tolerated by all patients. The time needed to place the clips was estimated to be no longer than an ordinary flexible bronchoscopy with biopsies. Although one clip loosened immediately after placement, it could be removed using biopsy forceps. No other complications occurred. As can be seen in figure 1, the clips are radiographically identifiable. The clips were used for delineation of the clinical target volume on the treatment planning system (fig. 1). In addition, the clips served as beacons facilitating the correct positioning of the patient with conebeam computed tomography (CT) during hypofractionated radiation therapy $(8 \times 7.5$ Gy EBRT).

3-4 weeks after EBRT, the patients underwent another bronchoscopy to remove the endobronchial clips by means of biopsy forceps. In cases 1 and 4 , both clips were easily retrieved. In case 2, one of the clips was missing, and in case 3 both clips had disappeared. Thorough examination of all 